\title{
A Prospective Observational Study on Changes in Physical Activity, Quality of Life, and Self-efficacy in Perioperative Patients with Gastric Cancer
}

\author{
Masako Shomura $^{{ }^{*}}$, Haruka Okabe ${ }^{1}$, Satoshi Iwamoto ${ }^{1}$, Emi Nakagawa $^{2}$, Kenji Nakamura ${ }^{3}$, Kazuhito Nabeshima ${ }^{3}$, Atsuko \\ Otsuka $^{4}$, Asako Murakoshi ${ }^{5}$, Yukiko Dozono ${ }^{4}$ and Noriko Sakaguchi ${ }^{6}$ \\ ${ }^{1}$ Department of Nursing, Tokai University School of Health Sciences, 143 Shimokasuya, Isehara City, Kanagawa 259-1193, Japan \\ ${ }^{2}$ Department of Nursing, Tokai University Oiso Hospital, 21-1 Gakkyo, Naka District, Oiso City, Kanagawa 259-0198, Japan \\ ${ }^{3}$ Department of Surgery, Tokai University School of Medicine, 143 Shimokasuya, Isehara City, Kanagawa 259-1193, Japan \\ ${ }^{4}$ Department of Nursing, Tokai University Hospital, 143 Shimokasuya, Isehara City, Kanagawa 259-1193, Japan \\ ${ }^{5}$ Home Care Agency Kaedenokaze, 6-18-1 Asamizodai, Minami District, Sagamihara City, Kanagawa 252-0328, Japan \\ ${ }^{6}$ Graduate School of Health Sciences, Tokai University, 143 Shimokasuya, Isehara City, Kanagawa 259-1193, Japan
}

\begin{abstract}
Background: Patients undergoing major surgery often experience "post-operative dysfunction", which may affect their physical strength and mental activity for a significant period post-operatively. We aimed to clarify the post-operative changes in physical activity, quality of life, and self-efficacy in patients with gastric cancer.

Methods: Physical activity, health-related quality of life, and self-efficacy were assessed in subjects using self-administered questionnaires before surgery, at discharge, and 1, 3, and 6 months after discharge. Post-operative parameters were compared to baseline data using the Friedman test with Bonferroni correction, as well as the Wilcoxon two-sample test.

Results: Seventeen subjects were included. Dietary intake and number of steps walked significantly decreased for up to 1 month after discharge, whereas physical activity of $\geq 3$ metabolic equivalents of task and exercise-expended energy were lowest at discharge. These differences were all statistically significant. Importantly, the patients did subsequently improve. Body mass index did not differ significantly at discharge, but showed a gradual, significant decrease at 1 and 3 months after discharge. With respect to health-related quality of life, the physical, role, and social functions decreased significantly for a month after surgery, whereas symptoms of fatigue, anorexia, and diarrhea were significantly severe during the first month after discharge. Self-efficacy in terms of symptoms, activities of daily living, and disease status significantly decreased at discharge, but subsequently improved.

Conclusions: Patients with gastric cancer showed lower dietary intake and body mass index up to 1 and 3 months after discharge, respectively. Physical activity and self-efficacy were lowest at discharge but improved subsequently. It is necessary, both preoperatively and in the first month following discharge, to provide patients with support that encourages self-management of physical changes and symptoms, and a return to normal levels of physical activity.
\end{abstract}

\section{Introduction}

Gastric cancer is the fourth most common malignancy, and the second leading cause of cancer deaths worldwide [1]. In Japan, by contrast, gastric cancer is the most common malignancy and the second leading cause of cancer deaths [2]. Treatment outcomes for gastric cancer are markedly improving; leading to longer postgastrectomy survival [3]. Despite recent advances in medical technology, a patient's clinical course post-gastrectomy is commonly complicated by a variety of symptoms collectively referred to as "postoperative dysfunction". Although the specific symptoms and severity vary, patients require approximately one year of convalescence before they regain their physical strength and mental stability [4]. Physical activity promotes health and prevents disease [5]. Indeed, physical activity may even be important for cancer prevention, although most studies have focused on the physical activity of patients with breast or colorectal cancers, which are common in Europe and the United States [6,7]. In Japan, several studies have documented a decrease in physical activity following surgery for esophageal cancer, with patients eventually recovering their physical functions (PFs) [8,9] Previous research has suggested that both self-efficacy and healthrelated quality of life (HRQOL) are associated with improved physical activity in breast cancer survivors $[10,11]$. We believe that the same may be true after gastric cancer surgery, although this has not been studied directly. The aim of this study was to identify changes in physical activity, HRQOL, and self-efficacy following cancer-related gastrectomy.

\section{Materials and Methods}

\section{Study design}

We conducted a prospective, longitudinal, observational study of perioperative patients with gastric cancer at two university hospitals

"Corresponding Author: Dr. Masako Shomura, RN, PhD, Department of Nursing, Tokai University School of Health Sciences, 143 Shimokasuya, Isehara City, Kanagawa 259-1193, Japan; E-mail: s-masako@tokai-u.jp

Citation: Shomura M, Okabe H, Iwamoto S, Nakagawa E, Nakamura K, et al. (2017) A Prospective Observational Study on Changes in Physical Activity, Quality of Life, and Self-efficacy in Perioperative Patients with Gastric Cancer. Int J Nurs Clin Pract 4: 219. doi: https://doi.org/10.15344/2394-4978/2017/219

Copyright: (C) 2017 Shomura et al. This is an open-access article distributed under the terms of the Creative Commons Attribution License, which permits unrestricted use, distribution, and reproduction in any medium, provided the original author and source are credited. 
Citation: Shomura M, Okabe H, Iwamoto S, Nakagawa E, Nakamura K, et al. (2017) A Prospective Observational Study on Changes in Physical Activity, Quality of Life, and Self-efficacy in Perioperative Patients with Gastric Cancer. Int J Nurs Clin Pract 4: 219. doi: https://doi.org/10.15344/2394-4978/2017/219

in a Japanese metropolitan area. Demographic and clinical assessments were performed at baseline. Using self-administered questionnaires, we investigated the subjects' physical activity levels, perioperative clinical changes, HRQOL, self-efficacy, and symptoms. The questionnaires were completed at baseline (before surgery), at discharge (within 2 weeks of surgery), and at 1,3 , and 6 months after discharge. This study was approved by the ethics committee of the Department of Health Science, Tokai University, Isehara, Japan, as well as by the institutional review boards of Tokai University Hospital, Isehara, Japan (ethics approval No.15R213). Written informed consent was obtained from all participants in the study.

\section{Conceptual framework}

The three main variables in this study were physical activity, selfefficacy, and HRQOL, which may not be independent of each other We hypothesized that self-efficacy and HRQOL contribute to the recovery of physical activity after surgery in patients with gastric cancer. Conversely, recovery of physical activity may contribute to improvements in self-efficacy and HRQOL (Figure 1).

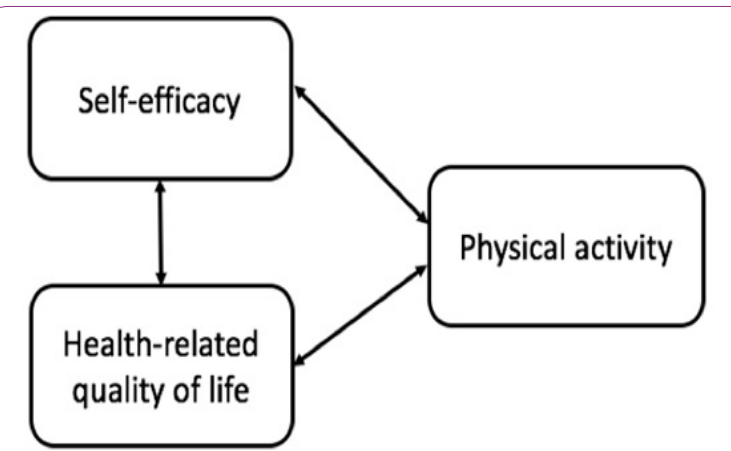

Figure 1: Conceptual framework of the study in patients with gastric cancer after surgery. Arrows indicate the direction of effect; rectangular figures indicate the main variables of this study.

\section{Patients}

We identified 54 candidates from which we recruited 25 subjects who met the inclusion and exclusion criteria. Patients were included if they (1) were aged 20 years or older; (2) were undergoing gastrectomy to treat incipient gastric cancer; (3) had prior knowledge of their gastric cancer; (4) had no other cancers during treatment; (5) had no serious mental illness or cognitive dysfunction; (6) were able to communicate in Japanese; and (7) were physically able to complete the 30-minute questionnaires. Patients were excluded if they (1) had heart, lung, cerebrovascular, nerve, or muscle diseases; (2) had restricted physical activity for medical reasons; or (3) were physically and mentally unable to participate in the study, as determined by their attending physician. Study withdrawal was permitted (1) at the patient's request, (2) if the patient experienced serious post-surgical complications (serious organ dysfunction requiring admission to the intensive care unit or suture failure/wound infection requiring repeat surgery), or (3) when orientation or cognitive function was markedly decreased after surgery compared to baseline.

\section{Clinical data collection}

Demographic and clinical data were collected at baseline using self administered questionnaires and electronic records. The following demographic data were collected: age, sex, and family structure, history of chronic diseases, occupation, and regular exercise levels. Perioperative data were also collected, including the clinical stage of gastric cancer, surgical technique (reconstruction), number of days to discharge, length of hospital stay, and post-operative adjuvant therapy. In addition, we recorded body mass index (BMI), dietary intake and number of meals per day, symptoms of dysfunction after gastric surgery, discomfort, and laboratory data such as hemoglobin levels.

The patients' weekly physical activity levels were measured using the number of steps taken per day, physical activity level equal to three or more metabolic equivalents of task (METs), and the amount of energy used in exercise. These parameters were measured using a physical activity meter (Kenz Lifecorder GS ; Suzuken, Nagoya, Japan) worn by patients for approximately 8 hours each day, and removed during bathing and sleeping. The data collected by the physical activity meters were analyzed using daily physical activity analysis software (Lifelyzer 05 Coach$^{\oplus}$; Suzuken, Nagoya, Japan). Specifically, we calculated the mean number of steps taken, METs, physical activity level at an intensity of $\geq 3$ METs, and activity duration and intensity.

\section{Measurements}

Physical activity, HRQOL, and self-efficacy were assessed using a self-administered questionnaire, which the patients completed at baseline, at discharge, and at 1, 3, and 6 months after discharge. The questionnaire addressed the following parameters: (1) physical activity, using the Japanese language edition (short version) of the International Physical Activity Questionnaire (IPAQ), (2) HRQOL, using the European Organization for Research and Treatment of Cancer QOL questionnaire version 3 (EORTCQLQ C-30v3), and (3) self-efficacy, using the Self-Efficacy Scale for Advanced Cancer (SEAC). Each questionnaire took approximately 30 minutes to complete, and patients completed the questionnaires at home and returned them by mail.

\section{Physical activity assessment}

The short version of IPAQ includes seven items related to exercise frequency, and measures physical activities $>3$ METs and energy expended (in kcal) in exercise per week $[12,13]$.

\section{HRQOL assessment}

The patients' HRQOL was measured using EORTCQLQ-C30v3, a questionnaire used by all patients with cancer [14]. It includes 30 questions examining symptoms, general health, and overall QOL, as well as questions relating to economic issues, and the five functional measures of QOL: physical functioning (PF2), role functioning (RF2), emotional functioning (EF), cognitive functioning (CF), and social functioning (SF). Scores are calculated individually and in several groups using prescribed methods. Each item receives a score between zero (minimum functionality and symptoms) and 100 (good functionality but maximum symptoms).

\section{Self-efficacy assessment}

Self-efficacy was measured using the SEAC, which comprises 18 items. The questionnaire is scored from zero to 100 , and higher scores indicate higher self-confidence on the part of the subject. It is currently used as a measure of self-efficacy in patients with cancer at all stages $[15,16]$.

\section{Statistical analysis}

All data were analyzed using the statistical package IBM SPSS Statistics version 23 for Windows (1989, Somers, NY, USA). Postoperative parameters were compared to baseline data using the 
Citation: Shomura M, Okabe H, Iwamoto S, Nakagawa E, Nakamura K, et al. (2017) A Prospective Observational Study on Changes in Physical Activity, Quality of Life, and Self-efficacy in Perioperative Patients with Gastric Cancer. Int J Nurs Clin Pract 4: 219. doi: https://doi.org/10.15344/2394-4978/2017/219

Page 3 of 6

Friedman test with Bonferroni correction, as well as the Wilcoxon twosample test. P-values $<0.05$ were considered statistically significant.

\section{Results}

\section{Patient characteristics}

The study included 17 subjects (12 men and 5 women), with an average age of 70 years. Of these, 10 subjects were stage I based on the tumor-node-metastasis (TNM) classification system, 16 underwent gastrectomy, 8 had full-time or part-time jobs, and 12 engaged in regular exercise (Table 1).

\begin{tabular}{|c|c|c|}
\hline Variables Age (years) & $\begin{array}{l}\text { Numbers of patient } \\
70.0 \pm 7.0(55.9-81.3)^{\mathrm{a}}\end{array}$ & \\
\hline \multirow[t]{2}{*}{ Gender } & Men & 12 \\
\hline & Women & 5 \\
\hline \multirow[t]{3}{*}{ Job status } & Full-time & 5 \\
\hline & Part-time & 3 \\
\hline & None & 9 \\
\hline \multirow[t]{2}{*}{ Regular exercise } & Yes & 12 \\
\hline & No & 4 \\
\hline \multirow[t]{2}{*}{ Cohabitant family members } & Yes & 16 \\
\hline & No & 1 \\
\hline \multirow[t]{5}{*}{ TNM stage } & IA & 10 \\
\hline & IB & 2 \\
\hline & IIB & 1 \\
\hline & IIIB & 3 \\
\hline & IV & 1 \\
\hline Post-operative hospital stay (days) & $\begin{array}{l}20.5 \pm 19.0(8-58) \\
{ }^{\text {a }} \text { Median } 10.5\end{array}$ & \\
\hline \multirow[t]{2}{*}{ Surgery } & Gastrectomy & 16 \\
\hline & Gastric bypass & 1 \\
\hline \multirow[t]{2}{*}{ Adjuvant } & S-1 & 3 \\
\hline & SP & 1 \\
\hline \multirow[t]{2}{*}{ Post-operative complications } & $\begin{array}{l}\text { Minor anastomotic } \\
\text { leakage }\end{array}$ & 3 \\
\hline & Pneumonia & 1 \\
\hline
\end{tabular}

Table 1: Patient characteristics at baseline $(\mathrm{N}=17)$.

Abbreviations: TNM, tumor node metastasis classification; S-1, tegafur/ gimeracil/oteracil potassium.

${ }^{a}$ Average value \pm SD (range: $\min$ - $\max$ ). Other values indicate numbers of patients.

\section{Physical conditions and physical activities}

The lowest BMI was observed 3 months after discharge (median $=20.7$ ), and remained significantly lower than baseline until at least 6 months after discharge (Figure 2A, Supplementary Tables 1 and 2).

The lowest median dietary intake occurred at the time of discharge (score $=3.0$ ). Dietary intake remained significantly lower than baseline for at least 6 months after discharge, but did increase significantly for 3 months after discharge, beyond which time there were no significant differences in dietary intake between time points (Figure 2a, Supplementary Tables 1 and 2).

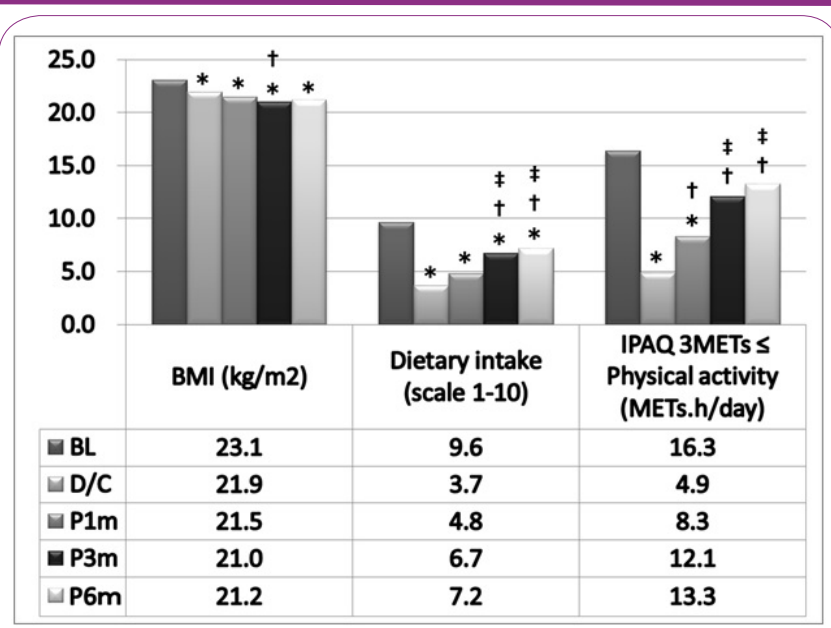

Figure 2a: Changes of BMI, intake, and 3 METs physical activity.

*; statistically significant compared with baseline (BL).

†; statistically significant compared with discharge (D/C).

‡; statistically significant compared with 1 month after discharge (P1m).

Abbreviations: $\mathrm{P} 3 \mathrm{~m} ; 3$ months after discharge, $\mathrm{P} 6 \mathrm{~m} ; 6$ months after discharge, BMI; body mass index; IPAQ, International Physical Activity Questionnaire; METs, metabolic equivalents

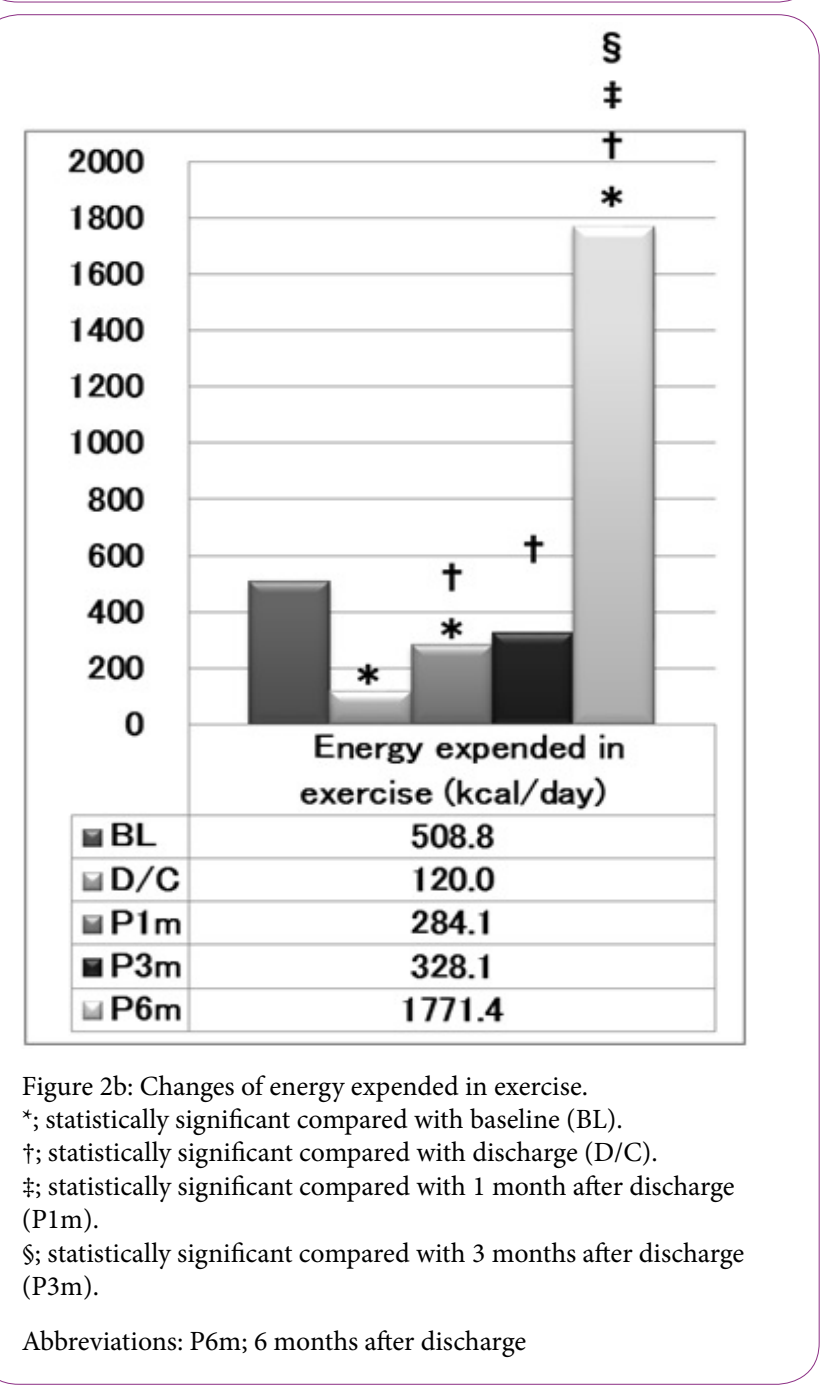


Citation: Shomura M, Okabe H, Iwamoto S, Nakagawa E, Nakamura K, et al. (2017) A Prospective Observational Study on Changes in Physical Activity, Quality of Life, and Self-efficacy in Perioperative Patients with Gastric Cancer. Int J Nurs Clin Pract 4: 219. doi: https://doi.org/10.15344/2394-4978/2017/219

Page 4 of 6

Physical activity $\geq 3$ METs was lowest at discharge, at 2.5 hours/day, and remained significantly lower than baseline for at least 1 month after discharge (Figure 2a, Supplementary Tables 1 and 2). Physical activity was significantly higher 6 months after discharge, although there were no significant differences between time points beyond 3 months after discharge. Energy used in exercise was lowest at discharge $(120.0 \mathrm{kcal} /$ day $)$, and increased significantly until at least 6 months after discharge (Figure $2 \mathrm{~b}$ and Supplementary Table 1 and 2).

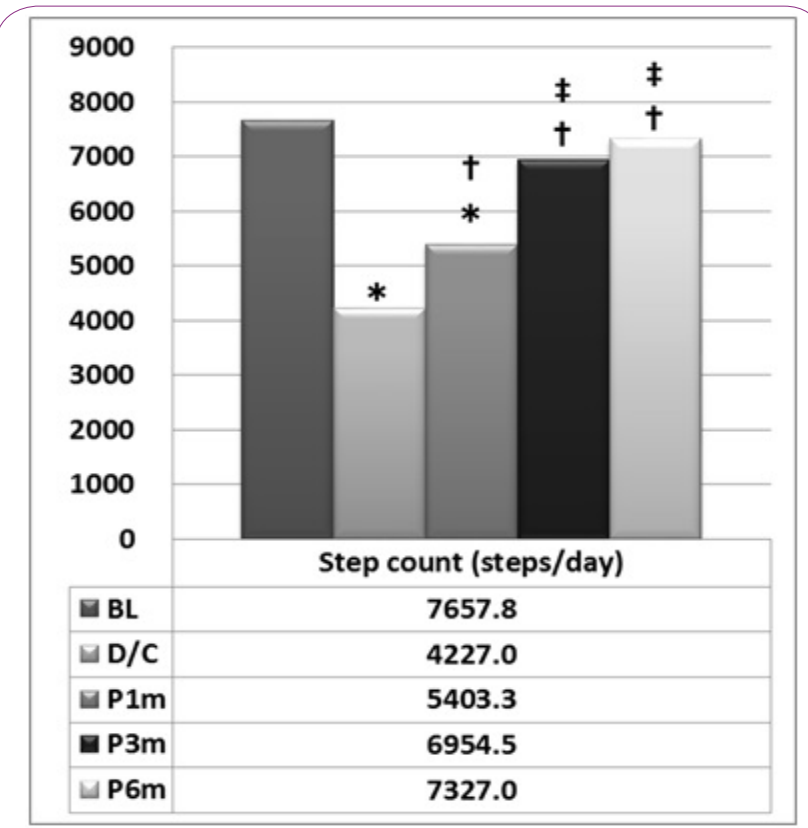

Figure 2c. Changes of step count.

*; statistically significant compared with baseline (BL).

$\dagger$; statistically significant compared with discharge (D/C).

\$; statistically significant compared with 1 month after discharge (P1m).

Abbreviations: $\mathrm{P} 3 \mathrm{~m} ; 3$ months after discharge, $\mathrm{P} 6 \mathrm{~m} ; 6$ months after discharge
The number of steps taken per day was lowest at discharge $(3,743)$ and began to increase at 1 month following surgery (Figure $2 c$, and Supplementary Table 1 and 2).

Serum hemoglobin levels were at their lowest at discharge (119 $\mathrm{g} / \mathrm{L}$ ), and showed significant increases until at least 6 months after discharge (Supplementary Table 1). Serum albumin was also at its lowest at discharge $(32 \mathrm{~g} / \mathrm{L})$ and increased gradually beyond 1 month after discharge $(\mathrm{P}<0.05)$, although no significant changes were observed beyond 3 months following discharge (Supplementary Table 2).

\section{HRQOL}

PF2, RF2, and SF, were all significantly lower than baseline at the time of discharge, as well as 1 month after surgery; however, all three measures improved significantly thereafter. None of the HRQOL scores differed significantly between 3 and 6 months after discharge, except for PF2 (Figure 3a, and Supplementary Tables 3 and Table 4). Symptoms of pain, dyspnea, and loss of appetite were significantly worse than baseline at the time of discharge and at 1 month after discharge. In addition, fatigue was lowest at discharge, and diarrhea worsened significantly at 3 months after discharge, though all symptom scores improved thereafter. There were no significant differences in sleeping disorders among any of the time points (Figure 3b, Supplementary Table 4).

\section{Self-efficacy}

We found significant differences in affect regulation efficacy (ARE), symptom coping efficacy (SCE), activities of daily living efficacy (ADE), and total efficacy (SE, total scores) between the pre-operative and all post-operative time points (Figure 4, Table 3 and Table 4). In particular, ARE and SE were lowest at discharge and within 1 month of discharge, but improved significantly thereafter. SCE was also significantly lowest at discharge (65.0), with significant improvement thereafter (Figure 4, Supplementary Tables 3 and 4). ADE was lowest

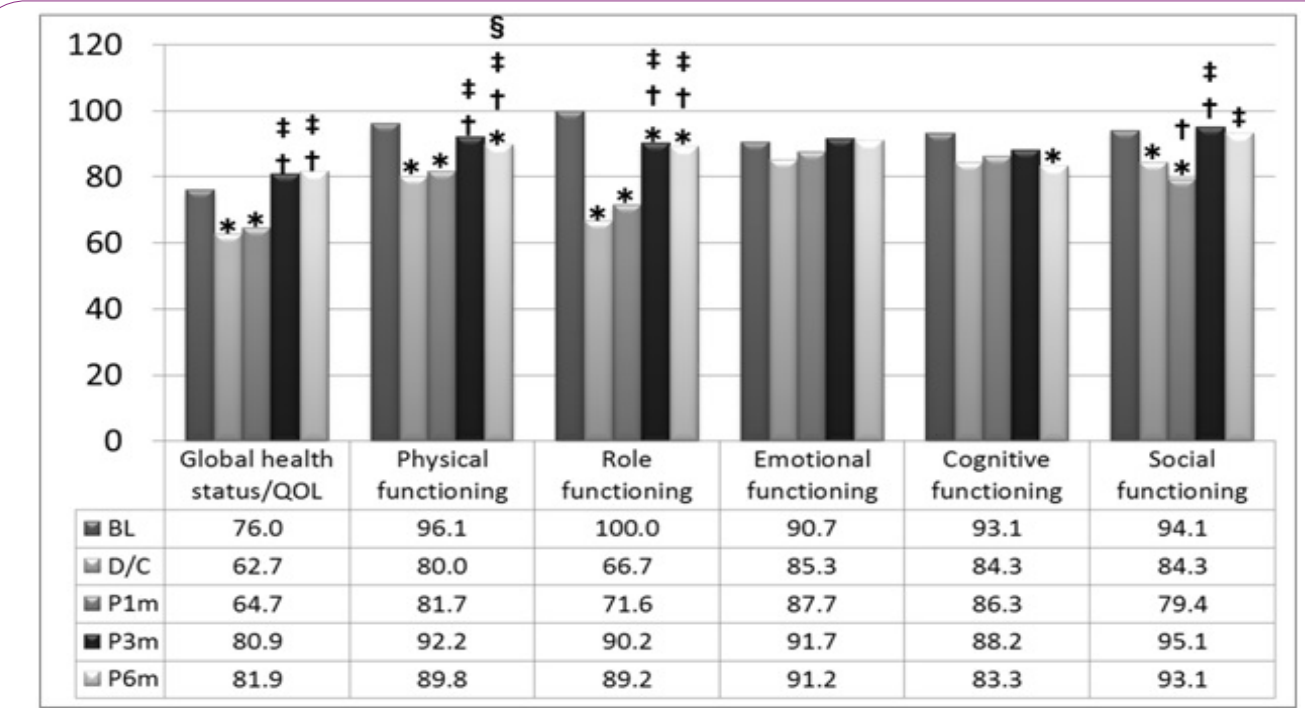

Figure 3a: Changes of health-related quality of life (global and functional aspect).

*; statistically significant compared with baseline (BL).

$\dagger$; statistically significant compared with discharge (D/C)

$\ddagger$; statistically significant compared with 1 month after discharge (P1m).

$\$$; statistically significant compared with 3 months after discharge (P3m).

Abbreviations: P6m; 6 months after discharge 
Citation: Shomura M, Okabe H, Iwamoto S, Nakagawa E, Nakamura K, et al. (2017) A Prospective Observational Study on Changes in Physical Activity, Quality of Life, and Self-efficacy in Perioperative Patients with Gastric Cancer. Int J Nurs Clin Pract 4: 219. doi: https://doi.org/10.15344/2394-4978/2017/219

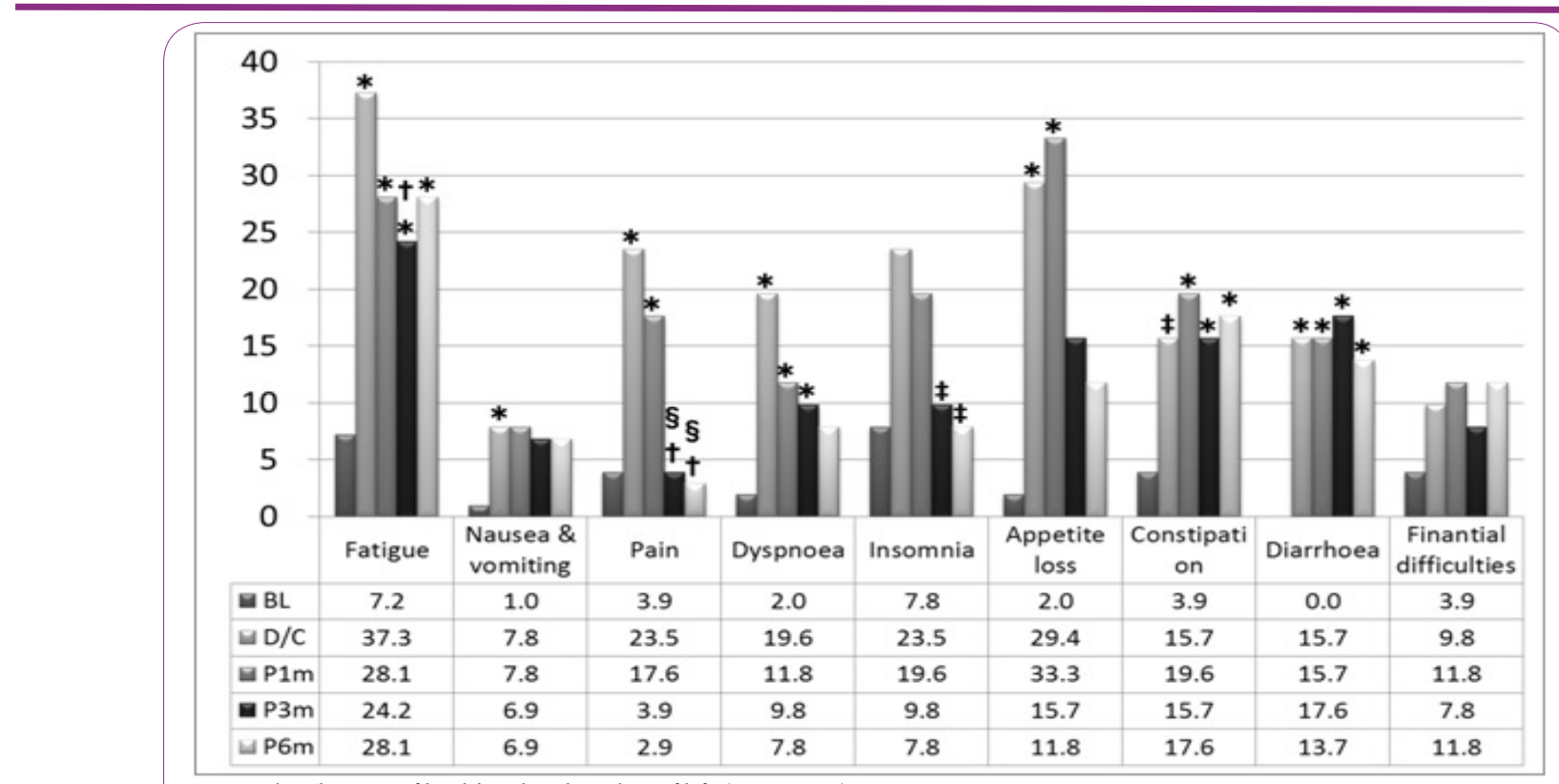

Figure 3b: Changes of health-related quality of life (symptoms).

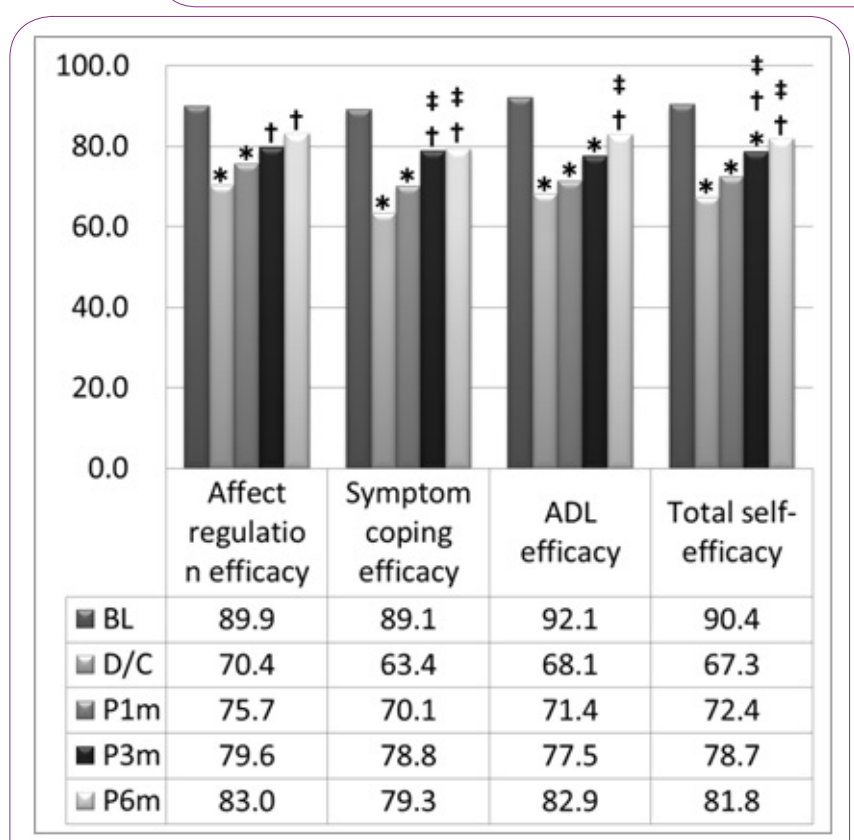

Figure 4: Changes of self-efficacy.

*; statistically significant compared with baseline (BL).

$\dagger$; statistically significant compared with discharge (D/C).

\$; statistically significant compared with 1 month after discharge (P1m).

Abbreviations: P3m; 3 months after discharge, P6m; 6 months after

discharge; ADL, activities of daily living

at 1 month after discharge and improved thereafter, and this finding was significant. No significant differences were found for any of these scores at 3 and 6 months after discharge (Figure 4, and Supplementary Table 4).

\section{Discussion}

Our investigation into physical condition and physical activity showed that dietary intake; IPAQ scores for physical activity of $\geq 3$ METs, energy expended in exercise, serum hemoglobin, and serum albumin were lowest at discharge. The patients' BMI showed significant gradual post-operative decreases until 3 months after discharge, whereas dietary intake and physical activity began to improve 1 month after surgery. The changes in BMI observed in this study are consistent with previous research by Kobayashi et al. [14], which found that patients' nutritional status is stable 3 months after surgery. In addition, 6 months after discharge, energy expended in exercise increased compared to that observed at baseline, suggesting a conscious effort to increase exercise after surgery. Laboratory parameters showed improvement earlier than in previous studies, which found that the lowest levels occur at 1 or 3 months after surgery $[17,18]$.

Our investigation into HRQOL indicated that the functional parameters PF, RF, and SF, as well as symptoms of pain, dyspnea, and appetite loss, were significantly lower at discharge and at 1 month post-discharge, compared to baseline. Physical function and activity, as well as HRQOL, dietary intake, hemoglobin, and albumin were all at their lowest at discharge. These findings were consistent with the scores of appetite loss and suggest that poor appetite at discharge continues for approximately 1 month. At discharge, registered dieticians provided nutritional guidance; however, it remains important to assess the patients' adherence to this guidance, as well as the nutritional consultation needs of patients whose dietary intake and laboratory data have not improved by 1 month after discharge. We found that physical activity, as measured by step counts, exercise related METs $\geq 3.0$, and the amount of energy expended in exercise, was at its lowest at discharge but improved at subsequent time points. $\mathrm{PF}, \mathrm{RF}$, and SF, as well as pain and dyspnea, were also at their lowest at discharge and 1 month after discharge, suggesting that physical activity returned to normal levels 1 month following discharge, despite dietary intake not having returned to normal levels. This probably led to functional problems, pain, and slight shortness-of-breath, among other symptoms. Our study subjects performed relatively satisfactory amounts of exercise. We believe that encouraging physical activity, mainly in the form of active walking, is important in increasing a patient's chances of recovering all physical functions and preventing loss of skeletal muscle mass, and that this regimen should start before 
Citation: Shomura M, Okabe H, Iwamoto S, Nakagawa E, Nakamura K, et al. (2017) A Prospective Observational Study on Changes in Physical Activity, Quality of Life, and Self-efficacy in Perioperative Patients with Gastric Cancer. Int J Nurs Clin Pract 4: 219. doi: https://doi.org/10.15344/2394-4978/2017/219

Page 6 of 6

surgery. Furthermore, it is crucial that clinicians consider patients BMI, dietary intake, laboratory data, and all other indicators of health.

Our investigation showed that self-efficacy, as measured by SCE symptoms, was lowest at discharge, and we believe that decreases in SCE led to HRQOL symptoms being at their lowest score at discharge. Although physical activity was at its lowest at discharge and improved thereafter, self-efficacy was at its lowest 1 month after discharge, suggesting that patients continue to experience intense anxiety even during recovery. It is important to assess physical activity 1 month after discharge to identify causes of poor improvement and to encourage exercise. Overall self-efficacy scores were at their lowest 1 month after discharge; for this reason, we believe that patients must be made aware of their own capabilities, and support must be provided to restore a positive outlook. Such an approach is likely to increase self-efficacy.

This study has several limitations. It was conducted at only two facilities with a small number of subjects, limiting the extent to which the results can be generalized. A multivariate analysis of influencing factors should be carried out in a larger number of subjects, which would inform evidence-based care practices.

\section{Conclusion}

Post-gastrectomy patients experience their lowest dietary intake, activity level, HRQOL, and self-efficacy at discharge or 1 month after discharge, after which these parameters improve. Conversely, BMI and diarrhea symptoms continue to decline 3 months after discharge. Improvements in physical condition are coupled with a gain in confidence, thus improving patients' ability to manage their HRQOL symptoms and their decreased physical functions. Based on our findings, it is necessary, both before surgery and up to 1 month after discharge, to provide post-gastrectomy patients with support that encourages both self-management of physical changes and symptoms, and a return to normal levels of physical activity. These findings may contribute to improved post-operative self-confidence, with subsequent improvement in HRQOL and physical functioning. It is therefore necessary to provide support and encouragement to post-operative gastric cancer patients as they cope with physical changes and symptoms. Recovery of physical activity is also necessary.

\section{Competing Interests}

The authors declare that they have no competing interests.

\section{Author Contributions}

Shomura M, Okabe H, Iwamoto S, and Nakagawa E contributed equally to this study; Nakamura $\mathrm{K}$ and Nabeshima K performed the surgery on the patients and evaluated the efficacy of therapy; Shomura M, Okabe H, Otsuka A, Dozono Y, Murakoshi A, and Sakaguchi N collected patient data, performed the statistical analysis, and were involved in the writing and editing of the manuscript; Shomura M designed the study and provided financial support for this work.

\section{Ethical Approval}

The study was conducted in accordance with the Declaration of Helsinki. The study protocol was approved by the institutional review board at the study site before imitation of the study. Written informed consent was obtained from each participant before enrollment.

\section{Funding}

This study was funded by a Japan Society for the Promotion of Science (JSPS) KAKENHI Grant-in-Aid for Scientific Research (C) 25463445 which have been received by M. Shomura from 2012 to 2016.

\section{References}

1. Jemal A, Bray F, Center MM, Ferlay J, Ward E, et al. (2011) Global cancer statistics. CA Cancer J Clin 61: 69-90.

2. Matsuda A, Matsuda T, Shibata A (2013) Cancer incidence and incidence rates in Japan in 2008: a study of 25 population-based cancer registries for the Monitoring of Cancer Incidence in Japan (MCIJ) project. JPN J Clin Oncol 44: 388-396.

3. Japanese Gastric Cancer Association Registration Committee, Maruyama K, Kaminishi M, Hayashi K, Isobe Y, et al. (2006) Gastric cancer treated 1991 in Japan: data analysis of nationwide registry. Gastric Cancer 9: 5166.

4. Takiguchi S, Yamamoto K, Hirao M, Imamura H, Fujita J, et al. (2012) A comparison of postoperative quality of life and dysfunction after Billroth $\mathrm{I}$ and Roux-en-Y reconstruction following distal gastrectomy for gastric cancer: results from a multi-institutional RCT. Gastric Cancer 15: 198-205.

5. Lee IM, Shiroma EJ, Lobelo F, Puska P, Blair SN, et al. (2012) Effect of physical inactivity on major non-communicable diseases worldwide: an analysis of burden of disease and life expectancy. Lancet 380: 219-229.

6. Ballard-Barbash R, Friedenreich CM, Courneya KS, Siddiqi SM, McTiernan A, et al. (2012) Physical activity, biomarkers, and disease outcomes in cancer survivors: a systematic review. J Natl Cancer Inst 104: 815-840.

7. Campbell PT, Patel AV, Newton CC, Jacobs EJ, Gapstur SM (2013) Associations of recreational physical activity and leisure time spent sitting with colorectal cancer survival. J Clin Oncol 31: 876-885.

8. Tatematsu N, Ezoe Y, Tanaka E, Muto M, Sakai Y, et al. (2013a) Impact of neoadjuvant chemotherapy on physical fitness, physical activity, and health-related quality of life of patients with resectable esophageal cancer. Am J Clin Oncol 36: 53-56.

9. Tatematsu N, Park M, Tanaka E, Sakai Y, Tsuboyama T (2013b) Association between physical activity and postoperative complications after esophagectomy for cancer: a prospective observational study. Asian Pac J Caner P 14: 47-51.

10. Loprinzi PD, Cardinal BJ (2013) Self-efficacy mediates the relationship between behavioral processes of change and physical activity in older breast cancer survivors. Breast Cancer 20: 47-52.

11. Vallance JK, Lavallee CM, Culos-Reed NS, Trudeau MG (2012) Physical activity is associated with clinically important differences in health-related quality of life among rural and small-town breast cancer survivors. Support Care Cancer 20: 1079-1087.

12. Craig CL, Marshall AL, Sjöström M, Bauman AE, Booth ML, et al. (2003) International physical activity questionnaire: 12-country reliability and validity. Med Sci Sports Exerc 35: 1381-1395.

13. Murase N, Katsumura T, Ueda C, Inoue S, Shimomitsu T (2002) Validity and reliability of Japanese version of International Physical Activity Questionnaire. Journal of Health and Welfare Statistics 49: 1-9.

14. Kobayashi K, Takeda F, Teramukai S, Gotoh I, Sakai H, et al. (1998) A cross-validation of the European Organization for Research and Treatment of Cancer QLQ-C30 (EORTC QLQ-C30) for Japanese with lung cancer. Eur J Cancer 34: 810-815.

15. Hirai K, Suzuki Y, Tsuneto S (2001) Self-efficacy scale for terminal cancer Japanese Journal of Psychosomatic Medicine 41: 19-27.

16. Hirai K, Suzuki Y, Tsuneto S, Ikenaga M, Hosaka T, et al. (2002) A structural model of the relationships among self-efficacy, psychological adjustment, and physical condition in Japanese advanced cancer patients. Psychooncol11: 221-229.

17. Kinoshita T, Shibasaki H, Oshiro T, Ooshiro M, Okazumi S, et al. (2011) Comparison of laparoscopy-assisted and total laparoscopic Billroth-I gastrectomy for gastric cancer: a report of short-term outcomes. Surg Endosc 25: 1395-1401.

18. Kosuga T, Hiki N, Nunobe S, Noma H, Honda M, et al. (2014) Feasibility and nutritional impact of laparoscopy-assisted subtotal gastrectomy for early gastric cancer in the upper stomach. Ann Surg Oncol 21: 2028-2035. 